\title{
An Optimal Order Process for Solving Finite Element Equations
}

\author{
By Randolph E. Bank and Todd Dupont
}

\begin{abstract}
A $k$-level iterative procedure for solving the algebraic equations which arise from the finite element approximation of elliptic boundary value problems is presented and analyzed. The work estimate for this procedure is proportional to the number of unknowns, an optimal order result. General geometry is permitted for the underlying domain, but the shape plays a role in the rate of convergence through elliptic regularity. Finally, a short discussion of the use of this method for parabolic problems is presented.
\end{abstract}

1. Introduction. In this work, we discuss the iterative solution of the large sparse linear systems which arise in connection with finite element procedures for solving selfadjoint elliptic boundary value problems. We take as our prototype the Neumann problem

$$
-\nabla \cdot(a \nabla u)+b u=f \quad \text { in } \Omega, \quad \frac{\partial u}{\partial n}=0 \quad \text { on } \partial \Omega,
$$

where $\Omega$ is a polygonal domain in $\mathbf{R}^{2}$. (In Section 4, we relax this assumption and require only that $\partial \Omega$ be piecewise smooth.) We assume $a \in C^{1}(\bar{\Omega}), b \in C(\bar{\Omega})$ and that there exist positive constants $\underline{a}, \bar{a}, \underline{b}, \bar{b}$ such that

$$
\underline{a} \leqslant a(x) \leqslant \bar{a}, \quad \underline{b} \leqslant b(x) \leqslant \bar{b}, \quad \text { for } x \in \bar{\Omega} .
$$

Then there exists a unique weak solution $u \in H^{1}(\Omega)$ for all $f \in L^{2}(\Omega)$. (In Section 4 , we remark on the singular case, $b \equiv 0$.)

Most of our arguments are applicable, with only minor modifications, to the Dirichlet problem

$$
-\nabla \cdot(a \nabla u)+b u=f \quad \text { in } \Omega, \quad u=0 \text { on } \partial \Omega,
$$

and we comment on such extensions as they arise.

We describe a $k$-level iterative procedure for solving the linear equations arising from some finite element discretizations of (1.1). Our scheme involves a sequence of nested triangulations of $\Omega, \mathcal{T}_{j}, j \geqslant 1$, and corresponding finite-dimensional subspaces $\Re_{j}$. Our objective, of course, is to obtain an approximate solution on the finest triangulation. The basic idea underlying multi-level techniques is that, under certain circumstances, the solution of a problem on a given grid can be well approximated by a solution on a coarser grid. Since the coarser grids have (geometrically) fewer unknowns, a substantial savings in computational effort can result. We prove that our procedure asymptotically (as $k \rightarrow \infty$ ) requires only $O\left(N_{k}\right)$

Received November 17, 1977; revised March 24, 1980.

1980 Mathematics Subject Classification. Primary 65N30, 65F10.

(C) 1981 American Mathematical Society $0025-5718 / 81 / 0000-0003 / \$ 05.25$ 
computations to produce an $O\left(N_{k}^{-q}\right)$ accurate solution, where $N_{k}$ is the dimension of $\mathfrak{R}_{k}$ and $q$ is the "correct" exponent. This is an optimal order work estimate.

Our $k$-level scheme is related to the multi-grid techniques of Bakhvalov [1], Brandt [6], Fedorenko [11], [12], and Nicolaides [17] for the solution of finite difference equations. However, the tools made available through the finite element formulation afford simplification of the analysis. For example, nonrectangular meshes are treated without affecting the nature of the convergence results or their proof. Also, in the finite difference context, there are questions of interpolation between various grids; these issues never explicitly arise in our treatment of the finite element case, but are implicitly handled by the approximation properties of the spaces $\mathfrak{R}_{j}$.

During the preparation of this manuscript we became aware of similar independent work by Nicolaides [18] and Hackbusch [14], [15]. In particular, in [14] Hackbusch proves results similar to Theorem 1, and in [18] Nicolaides proves a result similar to Corollary 1 . Not only are the methods of proof used here different from those cited, but the procedure we treat is different in detail. Having seen their treatments, we feel ours is somewhat simpler.

The remainder of the paper is organized as follows. In Section 2, we define terms, establish notation, and state our assumptions. In Section 3, we define and analyze the $k$-level scheme for $C^{0}$-piecewise linear finite elements. In Section 4 , we consider extensions of our method to the singular Neumann problem, to nonpolygonal $\Omega$, and to piecewise polynomial spaces of higher degree. In Section 5 , we consider briefly one possible extension of the $k$-level scheme to parabolic problems. Once a parabolic problem has been discretized in time, it can be viewed as a sequence of elliptic problems, and in this section we analyze the rate of convergence as a function of the time discretization parameter $\Delta t$.

2. Preliminaries and Notation. We seek numerical approximations of the weak form of (1.1): Find $u \in H^{1}(\Omega)$ such that

$$
a(u, v)=(f, v) \text { for all } v \in H^{1}(\Omega),
$$

where

$$
a(u, v)=\int_{\Omega} a \nabla u \cdot \nabla v+b u v d x, \quad(f, v)=\int_{\Omega} f v d x .
$$

The spaces $H^{k}$ for $k$ a positive integer will be the usual Sobolev spaces equipped with the norms

$$
\|u\|_{k}^{2}=\sum_{|\beta|<k}\left\|D^{\beta} u\right\|_{0}^{2}=\sum_{|\beta|<k}\left(D^{\beta} u, D^{\beta} u\right) .
$$

The spaces $H^{k}$ for $k$ positive and nonintegral will be defined by interpolation and $H^{k}$ for $k$ negative will be defined as the dual of $H^{-k}$. Corresponding to the bilinear form, $a(\cdot, \cdot)$ is the energy norm denoted $\|u\| \|^{2}=a(u, u)$.

We assume a modest amount of elliptic regularity for the solution of (2.1). Specifically, we assume that there exists a constant $0<\alpha \leqslant 1$ such that, for all $f \in H^{\alpha-1}$, there exists a unique solution $u \in H^{1+\alpha}$ of (2.1) and

$$
\|u\|_{1+\alpha} \leqslant C(a, b, \Omega)\|f\|_{\alpha-1} .
$$


We now turn to the discrete approximation of (2.1). Let $\mathcal{T}_{j}, j \geqslant 1$, be a nested sequence of triangulations of $\Omega$, constructed as follows. Take $\mathcal{T}_{1}$ to be a fixed triangulation; for $T \in \mathcal{T}_{1}$, denote the diameter of $T$ by $h_{T}$ and let $h_{T} \cdot d_{T}$ denote the diameter of the inscribed circle for $T$. Define

$$
h_{1}=\max _{T \in \mathscr{T}_{1}} h_{T}, \quad \delta_{0}=\min _{T \in \mathscr{T}_{1}} d_{T}, \quad \delta_{1}=\min _{T \in \mathscr{T}_{1}} h_{T} / h_{1} .
$$

The constant $\delta_{0}$ is a measure of the shape regularity of the triangles in $\mathcal{T}_{1}$, and $\delta_{1}$ is a measure of their uniformity.

We now construct $\mathcal{T}_{j}$ for $j>1$ inductively as follows. For each $T \in \mathscr{T}_{j-1}$, construct four triangles in $\sigma_{j}$ by pairwise connecting the midpoints of the edges. Each triangulation $\mathcal{T}_{j}$ will have shape regularity and uniformity constants $\delta_{0}$ and $\delta_{1}$ and will have $h_{j} \equiv \max _{T \in \mathscr{T}_{j}} h_{T}=h_{1} 2^{1-j}$.

With each triangulation $\widetilde{T}_{j}$, we associate the $N_{j}$-dimensional space $\mathfrak{N}_{j}$ of $C^{0}$-piecewise linear polynomials. Since the triangulations are nested, we have $\mathfrak{K}_{j} \subset \mathfrak{M}_{j+1}, j \geqslant 1$. The spaces $\mathfrak{K}_{j}$ satisfy the following standard approximation property [4], [5], [16], [21]: If $u \in H^{s}, 1 \leqslant s \leqslant 1+\alpha$, then there exists $u_{j} \in \Re_{j}$ such that

$$
\left\|u-u_{j}\right\|_{0}+h_{j}\left\|u-u_{j}\right\|_{1} \leqslant C\left(\delta_{0}, \delta_{1}, \Omega\right) h_{j}^{s}\|u\|_{s} .
$$

The finite element approximation of (2.1) is: Find $u_{j} \in \mathscr{R}_{j}$ such that

$$
a\left(u_{j}, v\right)=(f, v) \text { for all } v \in \Re_{j} .
$$

In our multi-level procedure, we will have occasion to consider problems of the more general form: Find $z \in \mathfrak{N}_{j}$ such that

$$
a(z, v)=G(v) \text { for all } v \in \mathfrak{T}_{j},
$$

where $G$ is a linear functional.

Associated with each space $\mathfrak{R}_{j}$ are eigenvalues $\lambda_{i}^{(j)}$ and eigenvectors $\psi_{i}^{(j)}$, $1 \leqslant i \leqslant N_{j}$, satisfying

$$
a\left(\psi_{i}^{(j)}, v\right)=\lambda_{i}^{(j)}\left(\psi_{i}^{(j)}, v\right) \text { for all } v \in \Re_{j} .
$$

Without loss of generality, we can require

$$
\begin{gathered}
0<\lambda_{1}^{(j)} \leqslant \lambda_{2}^{(j)} \leqslant \cdots \leqslant \lambda \oint_{j}, \\
\left(\psi_{i}^{(j)}, \psi_{k}^{(j)}\right)=\delta_{i k}, \quad a\left(\psi_{i}^{(j)}, \psi_{k}^{(j)}\right)=\lambda_{i}^{(j)} \delta_{i k},
\end{gathered}
$$

where $\delta_{i k}$ is the Kronecker delta.

A simple homogeneity argument shows

$$
\lambda_{N_{j}}^{(j)} \leqslant C\left(a, b, \delta_{0}, \delta_{1}, \Omega\right) h_{j}^{-2} \text {. }
$$

(Hereafter, we will drop the superscripts on the $\lambda_{i}^{(j)}$ and $\psi_{i}^{(j)}$.) We associate with each space the discrete norms $\|\cdot\| \|_{s},-2 \leqslant s \leqslant 2$, defined as follows. For $\chi \in \mathfrak{N}_{j}$, $\chi=\sum_{i=1}^{N_{j}} c_{i} \psi_{i}$; and

$$
\|\| \chi \|_{s}^{2}=\sum_{i=1}^{N_{j}} c_{i} \lambda_{i}^{s}, \quad-2 \leqslant s<2 .
$$

Note that $\|x\|_{0}=\|\chi\|_{0}$ and $\|\chi \chi\|_{1}=\|x\| \|$ are the usual $L^{2}$ and energy norms.

It is also useful to associate with $\mathfrak{K}_{j}$ a symmetric, positive definite bilinear form $b_{j}(\cdot, \cdot)$, which is comparable to the $L^{2}$ inner product. In particular, we assume 
there is a constant $\beta$, independent of $h_{j}$, such that

$$
0<\beta^{-1} \leqslant \frac{b_{j}(v, v)}{(v, v)} \leqslant \beta \text { for all } v \in \mathfrak{T}_{j}, v \neq 0 .
$$

We define eigenvalues $\tilde{\lambda}_{i}^{(j)}=\tilde{\lambda}_{i}$ and eigenvectors $\tilde{\psi}_{i}^{(j)}=\tilde{\psi}_{i}, 1 \leqslant i<N_{j}$, by

$$
\begin{gathered}
a\left(\tilde{\psi}_{i}, v\right)=\tilde{\lambda}_{i} b_{j}\left(\tilde{\psi}_{i}, v\right) \quad \text { for all } v \in \mathfrak{N}_{j}, \\
0<\tilde{\lambda}_{1} \leqslant \tilde{\lambda}_{2} \leqslant \cdots \leqslant \tilde{\lambda}_{N_{j}}, \\
b_{j}\left(\tilde{\psi}_{i}, \tilde{\psi}_{k}\right)=\delta_{i k}, \quad a\left(\tilde{\psi}_{i}, \tilde{\psi}_{k}\right)=\tilde{\lambda}_{i} \delta_{i k} .
\end{gathered}
$$

It follows from (2.11) and (2.14) that

$$
\tilde{\lambda}_{N_{j}}=\max _{\substack{v \in \Re_{j} \\ v \neq 0}} \frac{a(v, v)}{b_{j}(v, v)} \leqslant \beta \max _{\substack{v \in \mathscr{N}_{j} \\ v \neq 0}} \frac{a(v, v)}{(v, v)} \leqslant C\left(a, b, \Omega, \delta_{0}, \delta_{1}, \beta\right) h_{j}^{-2} .
$$

If $\chi \in \mathfrak{T}_{j}$, then $\chi=\sum_{i=1}^{N_{j}} \tilde{c}_{i} \tilde{\psi}_{i}$, and we define

$$
\|x\|_{s, b}^{2}=\sum_{i=1}^{N_{j}} \tilde{c}_{i}^{2} \tilde{\lambda}_{i}^{s}, \quad-2<s<2 .
$$

Note that $\|x \mid\|_{1, b}=\|\| \chi \|$, and that $\|x\|_{0, b}$ is comparable to $\|\chi\|_{0}$; thus we expect \|\|$\chi \|_{s, b}$ to be comparable in some sense to $\|\chi\|_{s}$ for $0<s<1$. We make this precise in Lemma 1.

Lemma 1. Let $\|x\| \|_{s, b}$ be defined by (2.16). Then there exists a constant $C=$ $C\left(a, b, \delta_{0}, \delta_{1}, \Omega, \beta\right)$ such that, for $0 \leqslant s \leqslant 1$,

$$
C^{-1}\|\chi\|_{s} \leqslant\|\chi\|_{s, b} \leqslant C\|\chi\|_{s},
$$

for all $\chi \in \Re_{j}$.

Lemma 1 is proved in the Appendix.

3. The $k$-Level Iteration. In this section we define and analyze the $k$-level scheme for solving (2.7). This procedure is actually composed of two recursive processes. The overall process involves solving problems (2.7) sequentially for $j=1,2, \ldots$ The second process involves generating approximate solutions of more general problems like (2.8). This second process forms the foundation upon which the overall algorithm for solving (2.7) rests. Thus, we begin by inductively defining a single iteration of the $k$-level scheme for solving (2.8) with $j=k$ :

(a) If $k=1,(2.8)$ is solved directly.

(b) If $k>1$, one iteration of the $k$-level scheme takes a given initial guess $z_{0} \in \mathfrak{T}_{k}$ to a final guess $z_{m+1} \in \mathfrak{T}_{k}$ ( $m$ an integer to be determined later) as follows: For $1 \leqslant l \leqslant m, z_{l}$ is defined by

$$
\left(z_{l}-z_{l-1}, v\right)=\lambda_{N_{k}}^{-1}\left\{G(v)-a\left(z_{l-1}, v\right)\right\} \quad \text { for all } v \in \mathfrak{T}_{k} .
$$

Let $q \in \mathfrak{K}_{k-1}$ be the approximation of $\bar{q} \in \mathfrak{K}_{k-1}$ obtained by applying $p$ iterations of the $k-1$-level scheme to the residual equation

$$
a(\bar{q}, v)=G(v)-a\left(z_{m}, v\right) \equiv \bar{G}(v) \text { for all } v \in \mathfrak{R}_{k-1},
$$

starting from initial guess zero ( $p$ is an integer to be determined later). Then set

$$
z_{m+1}=z_{m}+q \text {. }
$$


Equation (3.1) is called a smoothing iteration. Its purpose is to damp components of the error which oscillate on the order of the mesh size in $\mathscr{T}_{k}$. Once these oscillations have been damped, the error can be well approximated by an element $\bar{q} \in \mathfrak{M}_{k-1}$, a space whose dimension $N_{k-1} \sim N_{k} / 4$. Thus, in (3.2), we approximately compute the elliptic projection of the error into $\mathfrak{N}_{k-1}$ using $p$ iterations of the $k-1$-level scheme applied to a problem of the form (2.8) with $j=k-1$.

Note that (3.1) requires the solution of a linear system involving the mass (Gram) matrix at each step. We shall see below that (3.1) can be replaced by other, computationally more attractive, iterations without affecting the character of our convergence results. The use of the mass matrix simplifies the initial analysis, since the convergence of (3.1) does not depend on the choice of basis. Also, our use of $\lambda_{N_{k}}$ in (3.1) is convenient but nonessential; any upper bound of the form (2.11) will suffice.

TheOREM 1. Let (2.4) hold and let $p>1$ be any integer. Then there exists a constant $0 \leqslant \gamma<1$, and an integer $m \geqslant 1$, both independent of $h_{j}$, such that if

$$
\|\bar{q}-q\|\left\|\gamma^{p}\right\| \bar{q} \| \mid
$$

then

$$
\left\|\left|z-z_{m+1}\right|\right\| \leqslant \gamma\left\|\mid z-z_{0}\right\| .
$$

Proof. Our proof is motivated by the work of Bakhvalov [1], Brandt [6], Fedorenko [11], [12], and Nicolaides [17] for finite difference equations. The result is also similar to one obtained independently by Hackbusch [14], [15]. Note that (3.4) is essentially an induction hypothesis asserting that the $k-1$-level scheme can reduce the error by a factor of $\gamma$ in each iteration.

We begin with (3.4). Define the error $\varepsilon_{l}$ by

$$
\varepsilon_{l}=z-z_{l}, \quad 0 \leqslant l \leqslant m+1 .
$$

Then, from (3.1), we have

$$
\left(\varepsilon_{l}, v\right)=\left(\varepsilon_{l-1}, v\right)-\lambda_{N_{k}}^{-1} a\left(\varepsilon_{l-1}, v\right), \quad 1 \leqslant l \leqslant m .
$$

Expanding $\varepsilon_{0}$ in terms of the eigenfunctions (2.9)-(2.10) as $\varepsilon_{0}=\sum_{i=1}^{N_{k}} c_{i} \psi_{i}$, we have, from (3.6),

$$
\varepsilon_{l}=\sum_{i=1}^{N_{k}} c_{i} \psi_{i}\left(1-\lambda_{i} / \lambda_{N_{k}}\right)^{l}, \quad 0<l \leqslant m .
$$

Thus, we have

$$
\left\|\varepsilon_{m}\right\| \leqslant\left\|\varepsilon_{0}\right\| .
$$

We shall analyze (3.2) in two steps. First, we estimate the effect of the recursion. From (3.2), we have

$$
a\left(\bar{q}-\varepsilon_{m}, v\right)=0 \text { for all } v \in \Re_{k-1} .
$$

Taking $v=\bar{q}$ in (3.9) shows

$$
\|\bar{q}\||\leqslant|\left\|\varepsilon_{m} \mid\right\| .
$$

Using the hypothesis (3.4), (3.8), and (3.10) yields

$$
\|\bar{q}-q\|\left|\leqslant \gamma^{p}\left\|\mid \varepsilon_{0}\right\| .\right.
$$


We are left with estimating the error in the elliptic projection (3.9). Now

$$
\left\|\bar{q}-\varepsilon_{m}\right\|^{2}=a\left(\bar{q}-\varepsilon_{m}, \bar{q}-\varepsilon_{m}\right)=-a\left(\bar{q}-\varepsilon_{m}, \varepsilon_{m}\right) \leqslant\left\|\bar{q}-\varepsilon_{m}\right\|_{1-\alpha}\left\|\varepsilon_{m}\right\|_{1+\alpha} .
$$

To estimate $\left\|\varepsilon_{m}\right\| \|_{1+\alpha}$, use (2.12) and (3.7) to see

$$
\left\|\varepsilon_{m}\right\|_{1+\alpha}^{2}=\sum_{i=1}^{N_{k}} c_{i}^{2} \lambda_{i}^{1+\alpha}\left(1-\lambda_{i} / \lambda_{N_{k}}\right)^{2 m} \leqslant \lambda_{N_{k}}^{\alpha}\|g(x)\|_{\mathcal{L} \infty[0,1]}\left\|\varepsilon_{0}\right\|^{2},
$$

where $g(x)=x^{\alpha}(1-x)^{2 m}$. A simple calculation shows the maximum of $g(x)$ on $[0,1]$ occurs at $x=\alpha \cdot(2 m+\alpha)^{-1}$. Thus, we have

$$
\left\|\left|\varepsilon_{m}\left\|_{1+\alpha} \leqslant C h_{k}^{-\alpha} m^{-\alpha / 2}\right\|\right| \varepsilon_{0}\right\|,
$$

where we have used (2.11).

To estimate $\left\|\mid \bar{q}-\varepsilon_{m}\right\|_{1-\alpha}$, we use a standard duality argument and Lemma 1. Let $\rho \in H^{\alpha-1}$ and $\eta \in H^{1+\alpha}$ satisfy

$$
a(\eta, v)=(\rho, v) \text { for all } v \in H^{1} .
$$

Taking $v=\bar{q}-\varepsilon_{m}$, we have, for any $\chi \in \mathfrak{N}_{k-1}$,

$$
\begin{aligned}
\left(\rho, \bar{q}-\varepsilon_{m}\right) & =a\left(\eta, \bar{q}-\varepsilon_{m}\right)=a\left(\eta-\chi, \bar{q}-\varepsilon_{m}\right) \\
& \leqslant C h_{k}^{\alpha}\|\eta\|_{1+\alpha}\left\|\bar{q}-\varepsilon_{m}\right\| \leqslant C h_{k}^{\alpha}\|\rho\|_{\alpha-1}\left\|\bar{q}-\varepsilon_{m}\right\|,
\end{aligned}
$$

where we have used (2.4). Using this and Lemma 1 yields

$$
\left\|\bar{q}-\varepsilon_{m}\right\|_{1-\alpha} \leqslant C\left\|\bar{q}-\varepsilon_{m}\right\|_{1-\alpha} \leqslant C h_{k}^{\alpha}\left\|\mid \bar{q}-\varepsilon_{m}\right\| .
$$

Finally from (3.3) and (3.11)-(3.14)

$$
\left\|\left|\varepsilon_{m+1}\right|\right\|\left|=\left\|\varepsilon_{m}-q\right\|\|\leqslant\| \bar{q}-q\|\|+\left\|\varepsilon_{m}-\bar{q}\right\|\right| \leqslant\left(\gamma^{p}+C m^{-\alpha / 2}\right)\left\|\mid \varepsilon_{0}\right\| .
$$

To complete the proof choose $\gamma$ such that $\gamma^{p}<\gamma / 2$ and then choose $m$ such that $\mathrm{Cm}^{-\alpha / 2}<\gamma / 2$. Clearly the choices of $\gamma$ and $m$ can be made independent of $h_{j}$. Using these bounds with (3.15) completes the proof of Theorem 1 .

Before proceeding to convergence results for the $L^{2}$ norm, we pause to make several remarks. First, (3.15) can be sharpened somewhat by noting

$$
\begin{aligned}
\left.\left\|\varepsilon_{m+1}\right\|\right|^{2} & =\left\|\varepsilon_{m}-q\right\|\left\|^{2}=\right\| \varepsilon_{m}-\bar{q}\left\|^{2}+\right\| \bar{q}-q \|^{2}+2 a\left(\varepsilon_{m}-\bar{q}, \bar{q}-q\right) \\
& =\left\|\varepsilon_{m}-\bar{q}\right\|^{2}+\|\bar{q}-q\|^{2},
\end{aligned}
$$

where we have used (3.9). This leads to

$$
\left\|\left|\varepsilon_{m+1}\left\|^{2} \leqslant\left(\gamma^{2 p}+C m^{-\alpha}\right)\right\|\right| \varepsilon_{0}\right\|^{2} .
$$

To obtain the smallest value of $\gamma$ for a fixed $m$, i.e., that value which satisfies

$$
\gamma^{2 p}+C m^{-\alpha}=\gamma^{2}
$$

we must determine the roots of a polynomial of degree $p$ in $\gamma^{2}$. It is easy to see that the root of interest is formally given by a power series expansion of the form

$$
\gamma^{2}=\sum_{j=0}^{\infty} \alpha_{j}\left(\frac{C}{m^{\alpha}}\right)^{j(p-1)+1}=\frac{C}{m^{\alpha}}+\left(\frac{C}{m^{\alpha}}\right)^{p}+p\left(\frac{C}{m^{\alpha}}\right)^{2 p-1}+\ldots
$$

To obtain results for the $L^{2}$ norm, we assume $H^{2}$ regularity of the solution, i.e., (2.4) holds for $\alpha=1$. Since $\Omega$ is a polygon, this assumption implies that $\Omega$ must be convex [13], [21]. 
COROLlary 1. Let the assumptions of Theorem 1 hold for $\alpha=1$. Then there exists a constant $0 \leqslant \gamma<1$ and an integer $m \geqslant 1$, independent of $h_{j}$, such that, if

$$
\|q-\bar{q}\|_{0} \leqslant \gamma^{p}\|\bar{q}\|_{0}
$$

then

$$
\left\|z-z_{m+1}\right\|_{0} \leqslant \gamma\left\|z-z_{0}\right\|_{0} .
$$

Proof. Corollary 1 is similar in character to results obtained by Nicolaides [18] for the $l^{2}$ norm. From (3.7) it is immediate that

$$
\left\|\varepsilon_{m}\right\|_{0} \leqslant\left\|\varepsilon_{0}\right\|_{0}
$$

The duality argument leading to (3.14) now shows

$$
\left\|\bar{q}-\varepsilon_{m}\right\|_{0} \leqslant C h_{k} \mid\left\|\bar{q}-\varepsilon_{m}\right\| .
$$

To obtain the analogue of (3.11) use (3.16), (3.18), (3.19) and (2.11) to see

$$
\begin{aligned}
\|\bar{q}-q\|_{0} & \leqslant \gamma^{p}\|\bar{q}\| \leqslant \gamma^{p}\left\{\left\|\bar{q}-\varepsilon_{m}\right\|_{0}+\left\|\varepsilon_{m}\right\|_{0}\right\} \leqslant \gamma^{p}\left\{C h_{k}\left\|\bar{q}-\varepsilon_{m}\right\|+\left\|\varepsilon_{0}\right\|_{0}\right\} \\
& \leqslant \gamma^{p}\left\{C h_{k} \lambda_{N_{k}}^{1 / 2}+1\right\}\left\|\varepsilon_{0}\right\|_{0} \leqslant C \gamma^{p}\left\|\varepsilon_{0}\right\|_{0} .
\end{aligned}
$$

Finally from (3.12), with $\alpha=1$, and (3.19)

$$
\left\|\bar{q}-\varepsilon_{m}\right\|_{0}^{2} \leqslant C h_{k}^{2}\left\|\bar{q}-\varepsilon_{m}\right\|^{2} \leqslant C h_{k}^{2}\left\|\bar{q}-\varepsilon_{m}\right\|_{0}\left\|\varepsilon_{m}\right\|_{2} .
$$

An argument similar to (3.13) shows

$$
\left\|\varepsilon_{m}\right\|_{2} \leqslant C h_{k}^{-2} m^{-1}\left\|\varepsilon_{0}\right\|_{0}
$$

Thus, from (3.20)-(3.22), we have

$$
\left\|\varepsilon_{m+1}\right\|_{0} \leqslant\|\bar{q}-q\|_{0}+\left\|\varepsilon_{m}-\bar{q}\right\|_{0} \leqslant C\left(\gamma^{p}+m^{-1}\right)\left\|\varepsilon_{0}\right\|_{0},
$$

and Corollary 1 follows.

In practice, we prefer to replace the mass matrix on the left-hand side of (3.1) in order to reduce the cost of the smoothing iteration. Let $b_{k}(\cdot, \cdot)$ be a symmetric, positive definite bilinear form satisfying (2.13). We replace (3.1) with the smoothing iteration

$$
b_{k}\left(z_{l}-z_{l-1}, v\right)=\tilde{\lambda}_{N_{k}}^{-1}\left\{G(v)-a\left(z_{l-1}, v\right)\right\} \quad \text { for all } v \in \Re_{k} .
$$

COROLlary 2. Let the hypotheses of Theorem 1 hold. Let $p>1$ be any integer. Then there exists a constant $0 \leqslant \gamma<1$, and integer $m \geqslant 1$ such that, if (3.4) holds for the iteration based on (3.23), (3.2) and (3.3), then (3.5) follows.

Proof. The analogues of (3.6)-(3.11) are immediate. The analogue of (3.12) is

$$
\left.\left\|\bar{q}-\varepsilon_{m}\right\|\right|^{2} \leqslant\left\|\bar{q}-\varepsilon_{m}\right\|_{1-\alpha, b}\left\|\varepsilon_{m} \mid\right\|_{1+\alpha, b} .
$$

The analogue of (3.13) is immediate, as is the analogue of (3.14) (using Lemma 1). The remainder of the proof is unchanged.

There are many possible choices for $b_{j}(\cdot, \cdot)$. For example, if we use the standard nodal basis, then $(2.13)$ will be satisfied by $b_{j}(\cdot, \cdot)$ corresponding to the diagonal of the mass matrix, allowing the use of an under-relaxed Jacobi scheme for smoothing.

We now consider the overall computational cost of the iteration (3.1)-(3.3). Let $F(N)$ be the cost function associated with reducing the error by a factor of $\gamma$ for a problem with $N$ unknowns. We assume $F(N)$ is nondecreasing for $N \geqslant N_{1}$. The 
cost of implementing (3.1), as some variant of (3.23), and (3.3) can be bounded by $C_{1} m N_{k}=C_{2} N_{k}$ where $C_{1} m=C_{2}$ is independent of $k$. The cost of the recursion (3.2) is $p F\left(N_{k-1}\right)$. Thus,

$$
F\left(N_{k}\right) \sim p F\left(N_{k-1}\right)+C_{2} N_{k} .
$$

Since $N_{k} \sim 4 N_{k-1}$, the solution of (3.25) yields

(3.26) $F\left(N_{k}\right) \sim\left\{\begin{array}{l}F\left(N_{1}\right)\left(\frac{N_{k}}{N_{1}}\right)^{\log p / \log 4}+\frac{C_{2} N_{1}}{1-p / 4}\left(\frac{N_{k}}{N_{1}}-\left(\frac{N_{k}}{N_{1}}\right)^{\log p / \log 4}\right), \quad p \neq 4, \\ F\left(N_{1}\right)\left(\frac{N_{k}}{N_{1}}\right)+\frac{C_{2} N_{k}}{\log 4} \log \left(\frac{N_{k}}{N_{1}}\right), \quad p=4,\end{array}\right.$ where $F\left(N_{1}\right)$ is the fixed cost of solving a problem on the coarsest grid [19].

From (3.26) we have asymptotically (as $k \rightarrow \infty)$

$$
F(N) \leqslant \begin{cases}C_{3} N, & p=2,3 \\ C_{3} N \log N, & p=4 \\ C_{3} N^{\log p / \log 4}, & p \geqslant 5\end{cases}
$$

The choices of most interest are clearly $p=2,3$, since these lead to an optimal order algorithm, in the sense that the cost of reducing the error by a fixed factor $\gamma$ is asymptotically no worse than proportional to the number of unknowns. This does not necessarily lead to an optimal order algorithm overall, however, since we may be interested in reducing the initial error by a factor of $h_{k}^{q}$ for some fixed value of $q$. The straightforward implementation of the $k$-level scheme would then require $O(N \log N)$ operations.

To avoid the appearance of the $\log N$ factor, and thus to obtain an overall optimal order algorithm, we need to apply recursion once more. We assume that the solutions of (2.7) satisfy

$$
\left\|u-u_{j}\right\| \leqslant \mathcal{K} h_{j}^{q}, \quad j \geqslant 1,
$$

where $\mathcal{K}$ is a constant independent of $h_{j}$, and $\|\cdot\|$ denotes any fixed norm, for example the energy or $L^{2}$ norm. Let $\tilde{u}_{j}, j \geqslant 1$, be approximate solutions of the discrete problems (2.7), generated as follows.

For $j=1$, (2.7) is solved directly to obtain $\tilde{u}_{1}$. For $j>1$, starting from initial guess $\tilde{u}_{j-1}$ apply $r$ iterations of the $j$-level scheme to (2.7) to obtain $\tilde{u}_{j}$ ( $r$ is an integer to be specified below). Suppose that each iteration of the $j$-level scheme reduces the $\|\cdot\|$ norm of the error by a factor of $\gamma$. Let $e_{j}=\left\|u_{j}-\tilde{u}_{j}\right\|$; then

$$
\begin{aligned}
e_{j} & \leqslant \gamma^{r}\left\|u_{j}-\tilde{u}_{j-1}\right\| \leqslant \gamma^{r}\left\{\left\|u_{j}-u\right\|+\left\|u_{j-1}-u\right\|+e_{j-1}\right\} \\
& \leqslant \gamma^{r} e_{j-1}+\gamma^{r} \mathcal{K}\left(1+2^{q}\right) h_{j}^{q},
\end{aligned}
$$

where we have used $h_{j}=h_{j-1} / 2$. The solution of the majorizing difference equation yields

$$
e_{j} \leqslant \mathcal{K} \delta h_{j}^{q}, \quad j \geqslant 1, \quad \delta=\frac{\gamma^{r}\left(1+2^{q}\right)}{\left(1-\gamma^{r} 2^{q}\right)},
$$

where we have assumed that (3.29) holds for $j=1$, and that $\gamma^{r} 2^{q}<1$. Note that the choice of $r$ is independent of $h_{j}$. Thus, from (3.28)-(3.29),

$$
\left\|u-\tilde{u}_{j}\right\| \leqslant\left\|u-u_{j}\right\|+e_{j} \leqslant(1+\delta) \mathcal{K} h_{j}^{q}, \quad j \geqslant 1 .
$$


The cost of computing $\tilde{u}_{k}$ is bounded by

$$
F\left(N_{1}\right)+r \sum_{j=2}^{k} F\left(N_{j}\right) \leqslant C_{3} \frac{4}{3} r N_{k}=C_{5} N_{k},
$$

where we have used (3.27) to bound $F\left(N_{j}\right)$. We summarize this result in

THEOREM 2. Let Theorem 1 hold and let $p=2$ or 3 . Let the $k$-level scheme be implemented as described above, and let $\delta$ be defined in (3.29). If (3.28) holds and

then

$$
\left\|u_{1}-\tilde{u}_{1}\right\| \leqslant \delta \mathcal{K} h_{1}^{q}
$$

(a) $\left\|u_{j}-\tilde{u}_{j}\right\| \leqslant \delta \mathcal{K} h_{j}^{q}, j \geqslant 2$,

(b) $\left\|u-\tilde{u}_{j}\right\| \leqslant(1+\delta) \mathcal{K} h_{j}^{q}, j \geqslant 1$,

(c) the cost of computing $\tilde{u}_{k}$ is bounded by $C_{5} N_{k}$ where $C_{5}$ is independent of $k$.

We conclude with several remarks about the Dirichlet problem (1.2). In most respects, the $k$-level scheme for (1.2) is similar to that for the Neumann problem. We define $\mathcal{T}_{j}$ as in Section 2. Let $\mathfrak{T}_{j} \subset H_{0}^{1}$ be the space of $C^{0}$-piecewise linear polynomials associated with $\mathcal{T}_{j}$ satisfying the Dirichlet boundary conditions. Then $\mathfrak{K}_{j-1} \subset \mathfrak{K}_{j}, j \geqslant 2$, as before. With this modification, (3.1)-(3.3) and the conclusions of Theorems 1-2 and the corollaries remain valid.

4. Extensions. In this section we consider several extensions of the $k$-level scheme of Section 3.

A. The Singular Neumann Problem. When the coefficient $b(x) \equiv 0$ in (1.1), then the problem becomes singular. For a solution to exist, the average value of the right-hand side must be zero, i.e.,

$$
(f, 1)=0 .
$$

If (4.1) is satisfied, then the solution of (2.1) is determined uniquely up to an additive constant. The unique member $u^{*}$ of the set of admissible solutions with smallest $L^{2}$ norm (the least squares solution) is characterized by satisfying (2.1) and having average value zero. Let $H^{1 *}=\left\{u \in H^{1} \mid(u, 1)=0\right\}$. Then $u^{*} \in H^{1 *}$ satisfies

$$
a\left(u^{*}, v\right)=(f, v) \text { for all } v \in H^{1 *}
$$

and $a(\cdot, \cdot)$ induces a norm on $H^{1 *}$ comparable to $\|\cdot\|_{1}$.

Let $\mathcal{T}_{j}$ and $\mathfrak{K}_{j}, j \geqslant 1$, be defined as in Section 2 , and let $\mathfrak{T}_{j}^{*}=\{u \in$ $\left.\mathfrak{R}_{j} \mid(u, 1)=0\right\}$. Clearly $\mathfrak{R}_{j}^{*} \subset H^{1 *}$ and $\mathfrak{K}_{j}^{*} \subset \mathfrak{M}_{j+1}^{*}, j>1$. Let $u_{j}^{*} \in \mathfrak{K}_{j}^{*}$ satisfy

$$
a\left(u_{j}^{*}, v\right)=(f, v) \text { for all } v \in \mathfrak{T}_{j}^{*}, j \geqslant 1 .
$$

We can apply the $k$-level scheme to (4.3) with $j=k$. Assume the analogue of (2.4) is satisfied for (4.2) as long as $f$ satisfies (4.1). To verify the analogue of the approximation property (2.6), let $u \in H^{s} \cap H^{1 *}$ and $z \in \mathfrak{M}_{j}$ satisfy

$$
\|z-u\|_{0}+h_{j}\|z-u\|_{1}=\inf _{\chi \in \mathscr{N}_{j}}\|\chi-u\|_{0}+h_{j}\|\chi-u\|_{1}<C h_{j}^{s}\|u\|_{s} .
$$

Let $z^{*}=z-(z, 1) /(1,1) \in \mathfrak{M}_{j}^{*}$. Then

$$
\begin{aligned}
\inf _{x \in \mathbb{R}_{j}^{*}}\|x-u\|_{0} & +h_{j}\|x-u\|_{1} \\
& \leqslant\left\|z^{*}-u\right\|_{0}+h_{j}\left\|z^{*}-u\right\|_{1} \leqslant C h_{j}^{s}\|u\|_{s}+C^{*}(\Omega)|(z, 1)| .
\end{aligned}
$$


But

$$
|(z, 1)|=|(z-u, 1)| \leqslant\|z-u\|_{0}\|1\|_{0},
$$

and the analogue of (2.6) is an immediate consequence of (4.4)-(4.5).

The proofs of the analogues of Theorems 1-2 are now straightforward.

In practice, we prefer to compute with the spaces $\mathfrak{K}_{j}$ rather than $\mathfrak{T}_{j}^{*}$. This presents no problems. The smoothing iteration (3.1) remains well defined. At level one we must solve singular but always consistent linear systems of the form (2.8) with $j=1$. If we augment (3.1)-(3.3) with

$$
(q, 1)=0
$$

we can always define a unique solution for the linear systems at level one. Furthermore, (4.6) implies $\left(z_{m+1}-z_{0}, 1\right)=0$, so that the overall process preserves average values.

B. More General Triangulations. Thus far we have insisted that the triangulations $\mathcal{T}_{j}$ for the $k$-level scheme be constructed in the precise fashion outlined in Section 3 and that $\Omega$ be polygonal. We now explore the consequences of allowing more general sequences of triangulations in a polygonal domain, and of allowing $\partial \Omega$ itself to become piecewise smooth.

A review of our arguments reveals that the characterization of the triangulations $\mathcal{T}_{j}$ were employed in essentially three ways:

(i) The quasi-uniformity of the triangulations is implicit in the bounds (2.11) and (2.15), and shape-regularity is needed for the approximation assumption (2.6) to hold.

(ii) The nesting of the triangulations allowed us to construct subspaces such that $\mathfrak{\Re}_{j-1} \subset \mathfrak{\Re}_{j}, j \geqslant 2$.

(iii) The fact that the ratios $h_{j} / h_{j+1}$ were bounded independent of $j$ was used in establishing (3.14).

While the conditions on $\Omega$ and $\mathcal{T}_{j}$ are sufficient to guarantee (i)-(iii), they are not necessary. For example, suppose $\Omega$ is polygonal and $\sigma_{1}$ is constructed as in Section 2. Construct $\mathcal{T}_{j}, j>1$, by taking each triangle in $\mathcal{T}_{j-1}$ and subdividing it into nine congruent triangles, rather than four, by connecting the trisection points for each edge as shown.

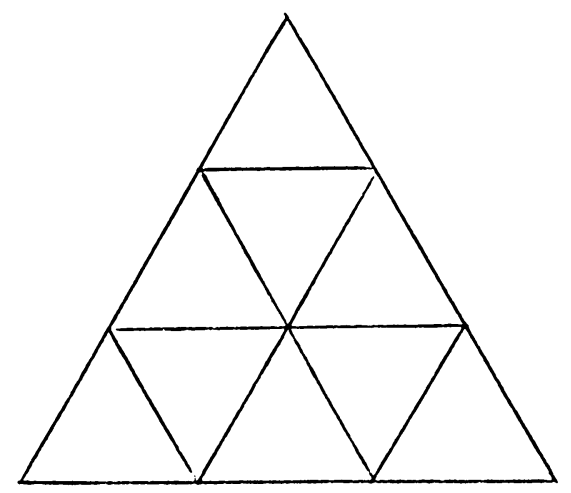

Let $\Re_{j}$ be the space of $C^{0}$-piecewise linear polynomials associated with $\mathcal{T}_{j}$. Then (i)-(iii) above are fulfilled, and the proofs of Theorems 1-2 go through without incident, except that $h_{j} / h_{j+1}=3$ rather than 2 . 
The operation counts are modified, however. Equation (3.25) remains valid, but now $N_{j} \sim 9 N_{j-1}, j>1$, so the asymptotic bound (3.27) is replaced by

$$
F(N) \leqslant \begin{cases}C_{3} N, & 2 \leqslant p \leqslant 8, \\ C_{3} N \log N, & p=9, \\ C_{3} N^{\log p / \log 9,} & p \geqslant 10 .\end{cases}
$$

Thus, the $k$-level scheme in this case remains of optimal order over a larger range of $p$.

One can generalize, and construct $\mathcal{T}_{j}$ by dividing each triangle in $\mathcal{T}_{j-1}$ into $l^{2}$ congruent triangles, and retain the results of Theorems $1-2$, as long as $l$ is independent of $h_{j}$. The crossover point in the analogue of (4.7) will then be $l^{2}$.

At this point, we are unsure of the optimal strategy or strategies regarding the construction of triangulations, in terms of minimizing the computational cost for problems of practical size. One can reach a given total refinement using fewer levels in the recursion, but the work per level will increase to maintain a comparable rate of convergence. On the other hand, one might settle for the slower rate of convergence for $l=3$ and simply carry out more (but less expensive) outer iterations to compensate.

Optimizing with respect to the parameters $p, m$, and now $l$, as well as with respect to the choice of the bilinear forms $b_{j}(\cdot, \cdot)$ to be used in (3.23) is an area for future research. We emphasize that such optimization occurs within a class of optimal order algorithms, and that one's concern may be directed toward some lower order terms, as well as the leading order terms in the operation count, for problems of practical size.

We now consider a $k$-level scheme for (1.1) when the boundary is piecewise smooth. We require each smooth piece of the boundary to have bounded curvature. The entire boundary should be Lipschitz. We allow triangles with one curved edge to be used near the boundary; the curved edge must be a smooth arc. We define the midpoint of a curved edge as the intersection of the edge with the perpendicular bisector of the chord joining the endpoints. (If $h_{1}$ is sufficiently small, this point is unique.)

Using this definition, we construct triangulations $\mathscr{T}_{j}, j \geqslant 1$, as in Section 2. As before, we define $h_{T}$ as the diameter of the circumscribing circle for $T \in \mathcal{T}_{j}$, and set $h_{j}=\max _{T \in T_{j}} h_{T}$. Note that in general $h_{j} \neq h_{1} 2^{1-j}$, although the ratios $h_{j} / h_{j+1}$ will remain bounded independent of $j$ (and will tend to two).

Let $\delta_{0}>0$ be a constant fixed independently of $h_{j}$. We require each triangle $T \in \mathcal{T}_{j}$ to be star-shaped with respect to each point in a circle of diameter $\delta_{0} h_{T}$ contained in $T$. For triangles with three straight edges, this is equivalent to our previous requirement that $\delta_{0} \leqslant d_{T}$. As before, we insure quasi-uniformity by requiring $h_{T} / h_{j} \geqslant \delta_{1}>0$ for all $T \in \mathcal{T}_{j}$, where $\delta_{1}$ is independent of $h_{j}$.

Let $\mathfrak{T}_{j}$ be the set of $C^{0}$-piecewise linear polynomials associated with $\mathcal{T}_{j}$. For triangles with three straight edges, local basis functions can be constructed in the usual way. For triangles with one curved edge, only a slight modification is needed. Suppose $T \in \mathcal{T}_{j}$ has one curved edge; define $T^{\prime}$ as a triangle with three straight edges given by the chord joining the endpoints of the curved edge and the two straight edges. The usual nodal basis functions, which are just linear polynomials, 
can be associated with $T^{\prime}$. We then extend each basis function as a linear polynomial to all of $R^{2}$ and then restrict it to $T$.

With this definition for $\mathscr{T}_{j}$ and $\mathfrak{N}_{j}$, requirements (ii) and (iii) above are trivially satisfied. The star-shaped requirement for triangles with curved edges can be used in conjunction with a recent version of the Bramble-Hilbert Lemma, due to Dupont and Scott [10], to establish (2.6) and hence (i). The proofs of Theorems 1-2 then follow as before.

We remark that the above constructions do not extend in straightforward fashion to the Dirichlet problem. The situation there is complicated by the fact that the appropriate trial spaces $\mathfrak{T}_{j}$ will not in general satisfy the boundary conditions exactly, but merely interpolate them at nodes lying on the boundary. See Scott [20] for a discussion of interpolated boundary conditions.

C. Polynomials of Higher Degree. We now extend the $k$-level scheme to spaces of $C^{0}$-piecewise polynomials of degree $s>1$. We assume (without loss of generality) that $\Omega$ is a polygon and that $\mathcal{T}_{j}, j \geqslant 1$, are constructed as in Section 2 . Let $\mathscr{T}_{j}$ denote the $N_{j}$-dimensional space of $C^{0}$-piecewise linear polynomials and $\mathfrak{R}_{j}$ denote the $\boldsymbol{M}_{j}$-dimensional space of $C^{0}$-piecewise polynomials of degree $s$ associated with $\mathscr{T}_{j}$. Note $\Re_{j} \subset \mathscr{K}_{j}, \Re_{j} \subset \mathscr{N}_{j+1}$, $\mathfrak{K}_{j} \subset \mathfrak{K}_{j+1}, j \geqslant 1$. Let $u_{j} \in \mathfrak{K}_{j}$ be the solutions of

$$
a\left(u_{j}, v\right)=(f, v) \text { for all } v \in \Re_{j}
$$

and let $z \in \mathfrak{M}_{j}$ satisfy

$$
a(z, v)=G(v) \text { for all } v \in \Re_{j},
$$

the analogues of (2.7)-(2.8). Define eigenvalues $\lambda_{i}$ and eigenvectors $\psi_{i}, 1<i<M_{j}$, using the analogues of (2.9)-(2.10). One iteration of the " $k+1$-level" scheme for solving (4.9), for $j=k$, (the analogue of (3.1)-(3.3)) involves the $k+1$ subspaces $\mathfrak{T}_{k}$ and $\mathfrak{T}_{j}, 1 \leqslant j \leqslant k$. This scheme takes initial guess $z_{0}$ to final guess $z_{n+1}$ as follows: For $1 \leqslant l \leqslant m, z_{l}$ is defined by

$$
\left(z_{l}-z_{l-1}, v\right)=\lambda_{M_{k}}^{-1}\left\{G(v)-a\left(z_{l-1}, v\right)\right\} \quad \text { for all } v \in \mathfrak{K}_{k} .
$$

We next compute $q \in \Re_{k}$, an approximation of $\bar{q} \in \Re_{k}$, using $p$ iterations of (3.1)-(3.3) applied to the linear system

$$
a(\bar{q}, v)=G(v)-a\left(z_{m}, v\right) \text { for all } v \in \Re_{k},
$$

starting from initial guess zero. Then set

$$
z_{m+1}=z_{m}+q \text {. }
$$

This scheme is quite similar to the $k$-level scheme of Section 3 , except that we have added a $(k+1)$ st special level in which the triangulation remains the same but the degree of the polynomials increases. All the requirements for the triangulations are satisfied, as is approximation property (2.6); thus the analysis of this scheme is virtually identical to Theorem 1.

The analysis of the computational complexity is slightly different. Let $F_{s}(M)$ be the positive, nondecreasing, cost function associated with reducing the initial error by a factor of $\gamma$ using (4.10)-(4.12). Then (3.25) is replaced by

$$
\begin{aligned}
F_{s}\left(M_{k}\right) & \sim p F_{1}\left(N_{k}\right)+C_{1} M_{k} ; \\
F_{1}\left(N_{j}\right) & \sim p F_{1}\left(N_{j-1}\right)+C_{2} N_{j}, \quad j>1 .
\end{aligned}
$$


The function $F_{1}(N)$ can be bounded as in (3.26)-(3.27), and this leads to a bound for $F_{s}(M)$, since $N_{k} \sim M_{k} r^{-2}$.

$$
F_{s}(M) \sim C_{3} M \text { for } p=2,3 .
$$

As in the piecewise linear case, we can obtain an optimal order algorithm for reducing the error by a factor of $h_{k}^{q}$ using a slight modification of the argument used in Section 3. Assume the solutions $u_{j}$ of (4.8) satisfy the analogue of (3.28) and let $\tilde{u}_{j}$ be approximate solutions generated as follows: For $j=1$ compute $\tilde{u}_{j}$ by direct solution or by a 2 -level scheme involving $\mathfrak{K}_{1}$ and $\mathfrak{N}_{1}$. (Somewhat better convergence results than those given here are obtainable for the 2-level scheme. See [2].) For $j>1$, start from $\tilde{u}_{j-1}$ and apply $r$ iterations of the $j+1$-level scheme (4.10)-(4.12) to obtain $\tilde{u}_{j}$.

For this scheme, the proof of the analogue of (3.29) is identical to that of Section 3 , and the conclusions of Theorem 2 remain valid.

5. Parabolic Problems. Consider the following problem: Find $z \in \mathfrak{T}_{j}$ such that

$$
\tau^{-1}(z, v)+a(z, v)=G(v) \text { for all } v \in \Re_{j},
$$

where $\tau>0$ is a positive constant. Problems of this type typically arise in solving parabolic partial differential equations which have been discretized in time $(\tau \sim$ $c \Delta t$, where $\Delta t$ is the time step) [3], [7], [8], [9]. We show that for $0<\tau<1$ the rate of convergence of the multi-level process is independent of $\tau$. This is intuitively reasonable because this problem should become easier to solve as $\tau \rightarrow 0$, since the mass matrix becomes dominant. In this section we analyze the convergence of the multi-level method for (5.1) as a function of the parameter $\tau$.

The principal importance of the result of this section is that it follows from Theorem 3 and the estimates of [3] and [7] that, under appropriate hypotheses on the parabolic problem, the approximate solution can be calculated in an amount of work that is proportional to the number of parameters that define it. Thus, we see that the optimal order work estimate proved for the multi-level process for elliptic problems carries over to parabolic problems.

Let triangulations $\mathcal{T}_{j}$ and spaces $\mathfrak{K}_{j}$ be defined as in Section 2 . Let $\lambda_{i}$ and $\psi_{i}$ be defined as in (2.9)-(2.11), and define, for $\chi \in \Re_{j}$,

$$
\|x\|_{1, \tau}^{2}=\|\| x\left\|_{1}^{2}+\tau^{-1}\right\| x \|_{0}^{2} .
$$

This is a natural norm in which to analyze the convergence of the multi-level scheme for (5.1).

The single $k$-level iteration for (5.1) can now be defined analogously to (3.1)(3.3) (for $k>1$ ). Let $z_{0} \in \mathfrak{N}_{k}$ be an initial guess. Define $z_{l} \in \mathfrak{N}_{k}, 1<l \leqslant m$, by

$$
\left(z_{l}-z_{l-1}, v\right)=\left(\lambda_{N_{k}}+\tau^{-1}\right)^{-1}\left\{G(v)-a\left(z_{l-1}, v\right)-\tau^{-1}\left(z_{l-1}, v\right)\right\}
$$

$$
\text { for all } v \in \mathfrak{M}_{k} \text {. }
$$

Let $q \in \mathfrak{K}_{k-1}$ be the approximation of $\bar{q} \in \mathfrak{R}_{k-1}$ obtained from $p$ iterations of the $k$ - 1-level scheme applied to

$$
a(\bar{q}, v)+\tau^{-1}(\bar{q}, v)=G(v)-a\left(z_{m}, v\right)-\tau^{-1}\left(z_{m}, v\right) \quad \text { for all } v \in \mathfrak{K}_{k-1} \text {. }
$$


Starting from initial guess zero, the set

$$
z_{m+1}=z_{m}+q \text {. }
$$

Theorem 3. Let (2.4) hold and let $p>1$ be any integer. Then there exists a constant $0 \leqslant \gamma<1$, and an integer $m \geqslant 1$, independent of $h_{k}$, such that if $0<\tau \leqslant 1$ and

$$
\|\bar{q}-q\|_{1, \tau} \leqslant \gamma^{p}\|\bar{q}\|_{1, \tau}
$$

then

$$
||\left|z_{m+1}-z\right|\left\|_{1, \tau} \leqslant \gamma \mid\right\| z_{0}-z \|_{1, \tau} .
$$

Proof. Let $\varepsilon_{l}=z-z_{l}, 0 \leqslant l \leqslant m+1$. Expanding $\varepsilon_{0}$ in terms of the eigenfunctions and using (5.2) leads to

$$
\varepsilon_{l}=\sum_{i=1}^{N_{k}} c_{i} \psi_{i}\left(1-\frac{\lambda_{i}+\tau^{-1}}{\lambda_{N_{k}}+\tau^{-1}}\right)^{l}=\sum_{i=1}^{N_{k}} c_{i} \psi_{i}\left(1-\frac{\lambda_{i}}{\lambda_{N_{k}}}\right)^{l}\left(1+\frac{1}{\tau \lambda_{N_{k}}}\right)^{-l},
$$

the analogue of (3.7). Thus,

$$
\left\|\varepsilon_{m}\right\|_{1, \tau} \leqslant\left(1+1 /\left(\tau \lambda_{N_{k}}\right)\right)^{-m} \mid\left\|\varepsilon_{0}\right\|_{1, \tau} .
$$

From (5.3), we have the analogue of (3.9)

$$
a\left(\bar{q}-\varepsilon_{m}, v\right)+\tau^{-1}\left(\bar{q}-\varepsilon_{m}, v\right)=0 \text { for all } v \in \mathfrak{T}_{k-1},
$$

from which it follows that

$$
\left\|\left|\bar{q}\left\|_{1, \tau} \leqslant\right\|\right|\left|\varepsilon_{m}\right|\right\|_{1, \tau} \leqslant\left(1+1 /\left(\tau \lambda_{N_{k}}\right)\right)^{-m}\left\|\left|\varepsilon_{0}\right|\right\|_{1, \tau} .
$$

The induction hypothesis (5.5) and (5.10) now yield

$$
\|\bar{q}-q\|_{1, \tau} \leqslant \gamma^{p}\left(1+1 /\left(\tau \lambda_{N_{k}}\right)\right)^{-m} \mid\left\|\varepsilon_{0}\right\|_{1, \tau}
$$

Obtaining a suitable bound for $\left\|\bar{q}-\varepsilon_{m}\right\|_{1, \tau}$ is slightly more complicated than in the proof of Theorem 1. For any $\chi \in \mathfrak{T}_{k-1}$,

$$
\left\|\bar{q}-\varepsilon_{m}\right\|_{1, \tau} \leqslant\left\|\chi-\varepsilon_{m}\right\|_{1, \tau} \leqslant\left(\lambda_{N_{k}}+\tau^{-1}\right)^{1 / 2}\left\|\chi-\varepsilon_{m}\right\|_{0} .
$$

Choose $\chi$ to be the $L^{2}$ projection of $\varepsilon_{m}$ into $\Re_{k-1}$; then

(5.13) $\left\|\chi-\varepsilon_{m}\right\|_{0}^{2}=\left(\chi-\varepsilon_{m}, \chi-\varepsilon_{m}\right)=-\left(\chi-\varepsilon_{m}, \varepsilon_{m}\right) \leqslant\left\|\chi \chi-\varepsilon_{m}\right\|\left\|_{-1-\alpha}\right\| \varepsilon_{m}\|\|_{1+\alpha}$.

To estimate the first term, we use a lemma, due to Thomée [22], (proved in the Appendix) to see

$$
\|\| \chi-\varepsilon_{m} \|_{-1-\alpha} \leqslant C\left\{\left\|\chi-\varepsilon_{m}\right\|_{-1-\alpha}+h_{k}^{2 \alpha}\left\|\chi-\varepsilon_{m}\right\|_{\alpha-1}\right\} .
$$

Now for $r=1 \pm \alpha$, we have, for any $\varphi \in \mathfrak{N}_{k-1}$,

$$
\left\|\chi-\varepsilon_{m}\right\|_{-r}=\sup _{v \in H^{r}} \frac{\left(\chi-\varepsilon_{m}, v\right)}{\|v\|_{r}}=\sup _{v \in H^{r}} \frac{\left(\chi-\varepsilon_{m}, v-\varphi\right)}{\|v\|_{r}}<C h_{k}^{r}\left\|\chi-\varepsilon_{m}\right\|_{0} .
$$

Thus, from (5.14)-(5.15),

$$
\left\|x-\varepsilon_{m}\right\|_{-1-\alpha} \leqslant C h_{k}^{1+\alpha}\left\|\chi-\varepsilon_{m}\right\|_{0} .
$$


Finally, to estimate \|\|$\varepsilon_{m} \|_{1+\alpha}$, we have

$$
\begin{aligned}
\left\|\varepsilon_{m}\right\|_{1+\alpha}^{2} & =\sum_{i=1}^{N_{k}} c_{i}^{2} \lambda_{i}^{1+\alpha}\left(1+\frac{\lambda_{i}}{\lambda_{N_{k}}}\right)^{2 m}\left(1+\frac{1}{\tau \lambda_{N_{k}}}\right)^{-2 m} \\
& =\lambda_{N_{k}}^{\alpha}\left(1+\frac{1}{\tau \lambda_{N_{k}}}\right)^{-2 m-1} \sum_{i=1}^{N_{k}} c_{i}^{2}\left(\lambda_{i}+\tau^{-1}\right)\left(1+\frac{\lambda_{i}}{\lambda_{N_{k}}}\right)^{2 m}\left(\frac{\lambda_{i}}{\lambda_{N_{k}}}\right)^{\alpha} \\
& \leqslant C \lambda_{N_{k}}^{\alpha} m^{-\alpha}\left(1+\frac{1}{\tau \lambda_{N_{k}}}\right)^{-2 m-1}\left\|\varepsilon_{0}\right\|_{1, \tau}^{2}
\end{aligned}
$$

Thus, from (5.12), (5.13), (5.16) and (5.17), we have

$$
\left\|\bar{q}-\varepsilon_{m}\right\|_{1, \tau} \leqslant C m^{-\alpha / 2}\left(1+1 /\left(\tau \lambda_{N_{k}}\right)\right)^{-m}\left\|\mid \varepsilon_{0}\right\|_{1, \tau},
$$

and from (5.11) and (5.18)

$$
\begin{aligned}
\left\|\varepsilon_{m+1} \mid\right\|_{1, \tau} & \leqslant\|\bar{q}-q\|_{1, \tau}+\|\| \varepsilon_{m}-\bar{q} \|_{1, \tau} \\
& \leqslant\left(1+\frac{1}{\tau \lambda_{N_{k}}}\right)^{-m}\left(\gamma^{p}+C m^{-\alpha / 2}\right)\left\|\varepsilon_{0}\right\|_{1, \tau} .
\end{aligned}
$$

This is analogous to (3.19) except for the presence of the "helping" term $\left(1+1 /\left(\tau \lambda_{N_{k}}\right)\right)^{-m}$. The remainder of the proof is standard.

If $\tau \sim c \Delta t$, then $1 /\left(\tau \lambda_{N_{k}}\right) \sim C h_{k}^{2} / \Delta t$. Therefore, if $\Delta t \leqslant O\left(h_{k}^{2}\right)$, the helping term is, by itself, sufficient to guarantee the result of Theorem 3, i.e., the smoothing iteration (5.2) reduces the error by a fixed amount independent of $h_{k}$ in each iteration. For $C^{0}$-piecewise polynomials of degree $s$, the analogue of Theorem 3 is true. If we discretize in time, using a second order correct in time scheme (for example Crank-Nicolson), then we would generally expect to have $\Delta t^{2}=O\left(h^{s+1}\right)$ [7], [8], [9]. Then, for the important cases of $s=1,2$ (piecewise linears and quadratics), the multi-level iteration (5.2)-(5.4) would be very useful. For $s \geqslant 3$, the multi-level iteration would still converge as predicted in Theorem 3 , but $\Delta t$ would then be sufficiently small that the smoothing iteration by itself would have been adequate. Higher order time discretizations [3] lead to similar results. For a $q$ th order time discretization $\Delta t^{q}=O\left(h^{s+1}\right)$, and the multi-level scheme is superior to smoothing for $s+1<2 q$.

\section{Appendix.}

(A) Proof of Lemma 1. As far as we know, the comparability of norms in Lemma 1 depends on the fact that all triangles are of approximately the same size, i.e., $\delta_{1} \leqslant h_{T} / h_{j}$ for $T \in \mathcal{T}_{j}$.

Let $P$ be the $L^{2}$ projection onto $\Re_{j}$. Considered as a map of $L^{2}(\Omega)$ onto $\Re_{j}$, equipped with the $L^{2}$ norm, $P$ is a bounded operator with norm 1 . Considered as a map of $L^{2}(\Omega)$ onto $\Re_{j}$, equipped with the $\|\cdot\|_{0, b}$ norm, $P$ is a bounded operator with norm bounded by $\beta^{1 / 2}$, since, from (2.13),

$$
\|P u\|_{0, b} \leqslant \beta^{1 / 2}\|P u\|_{0} \leqslant \beta^{1 / 2}\|u\|_{0} .
$$

Next, considered as a map from $H^{1}(\Omega)$ onto $\mathfrak{T}_{j}$, equipped with the energy (or $\left.\mid\|\cdot\|_{1, b}\right)$ norm, $P$ is a bounded operator with bound $C=C\left(\delta_{1}\right)$. To see this, note that for appropriate $v \in \mathfrak{T}_{j}$, 


$$
\begin{aligned}
\|P u\|_{1, b} & \equiv\|P u\| \leqslant\|P(u-v)\|+\|v\| \leqslant C h_{j}^{-1}\|P(u-v)\|_{0}+C\|v\|_{1} \\
& \leqslant C h_{j}^{-1} h_{j}\|u\|_{1}+C\|u\|_{1},
\end{aligned}
$$

where we have used (2.6).

If we interpolate between the latter two maps, the conclusion follows, since $\||\cdot|\|_{s, b}, 0<s<1$ are clearly the interpolation norms between $\mathfrak{M}_{j}$ equipped with the \|\|$\cdot \|_{0, b}$ norm and $\mathfrak{N}_{j}$ equipped with the energy norm.

This verifies the right-hand side of (2.17). That the inequality can be reversed follows by applying interpolation of operators to the injection map of $\Re_{j}$ into $L^{2}$ and $H^{1}$.

(B) Proof of (5.14). Let $\chi \in \mathfrak{T}_{k}$ be fixed and let $\eta_{h} \in \mathfrak{N}_{k}$ and $\eta \in H^{1}$ satisfy

$$
\begin{aligned}
& a\left(\eta_{h}, v\right)=(\chi, v) \quad \text { for all } v \in \mathfrak{N}_{k} \text {, } \\
& a(\eta, v)=(\chi, v) \quad \text { for all } v \in H^{1} \text {. }
\end{aligned}
$$

Note

$$
a\left(\eta_{h}-\eta, v\right)=0 \text { for all } v \in \pi_{k} .
$$

If $\chi=\sum_{i=1}^{N_{k}} c_{i} \psi_{i}$, then $\eta_{h}=\sum_{i{ }_{1}}^{N_{k}} c_{i} \lambda_{i}^{-1} \psi_{i}$ and

$$
\|x\|_{-1-\alpha}=\|\| \eta_{h}\|\|_{1-\alpha} \leqslant C\left\|\eta_{h}\right\|_{1-\alpha}
$$

by virtue of Lemma 1 . Now,

$$
\left\|\eta_{h}\right\|_{1-\alpha} \leqslant\|\eta\|_{1-\alpha}+\left\|\eta_{h}-\eta\right\|_{1-\alpha} \leqslant C\|x\|_{-1-\alpha}+\left\|\eta_{h}-\eta\right\|_{1-\alpha},
$$

where we have used (2.4). To estimate $\left\|\eta_{h}-\eta\right\|_{1-\alpha}$, we use duality. Let $\rho, \xi$ satisfy

$$
a(\rho, v)=(\xi, v) \quad \text { for all } v \in H^{1} .
$$

Taking $v=\eta_{h}-\eta$ and using (A.4), (2.4) and (2.6), we have,

$$
\begin{aligned}
\left(\xi, \eta-\eta_{h}\right) & =a\left(\rho-v, \eta-\eta_{h}\right) \leqslant C h_{k}^{\alpha}\|\rho\|_{1+\alpha}\left\|\eta-\eta_{h}\right\|_{1} \\
& \leqslant C h_{k}^{\alpha}\|\xi\|_{\alpha-1}\left\|\eta-\eta_{h}\right\|_{1}, \quad v \in \mathscr{N}_{k} .
\end{aligned}
$$

Thus,

$$
\left\|\eta-\eta_{h}\right\|_{1-\alpha} \leqslant C h_{k}^{\alpha}\left\|\eta-\eta_{h}\right\|_{1} \leqslant C h_{k}^{2 \alpha}\|\eta\|_{1+\alpha} \leqslant C h_{k}^{2 \alpha}\|x\|_{\alpha-1} .
$$

(5.14) now follows from (A.5)-(A.7).

Department of Mathematics

The University of Texas

Austin, Texas 78712

Department of Mathematics

University of Chicago

Chicago, Illinois 60637

1. N. S. Bakhvalov, "On the convergence of a relaxation method with natural constraints on the elliptic operator," Ż. Vyčisl. Mat.i Mat. Fiz., v. 6, 1966, pp. 861-885.

2. R. E. BANK \& TODD DUPONT, "Analysis of a two-level scheme for solving finite element equations," Numer. Math. (Submitted.)

3. J. H. Bramble, "Discrete methods for parabolic equations with time-dependent coefficients," Numerical Methods for PDEs, Academic Press, New York, 1979, pp. 41-52.

4. J. H. Bramble \& S. R. Hilbert, "Bounds for a class of linear functionals with applications to Hermite interpolation," Numer. Math., v. 16, 1971, pp. 362-369.

5. J. H. Bramble \& M. Zlamal, "Triangular elements in the finite element method," Math. Comp., v. 24, 1970, pp. 809-821. 
6. A. Brandt, "Multi-level adaptive solutions to boundary value problems," Math. Comp., v. 31, 1977, pp. 333-390.

7. Jim Douglas, JR., Todd Dupont \& R. E. Ewing, "Incomplete iteration for time-stepping a Galerkin method for a quasi-linear parabolic problem," SIAM J. Numer. Anal., v. 16, 1979, pp. 503-522.

8. Jim Douglas, JR. \& Todd Dupont, "Galerkin methods for parabolic equations," SIAM J. Numer. Anal., v. 7, 1970, pp. 575-626.

9. JiM Douglas, JR., "Effective time-stepping methods for the numerical solution of nonlinear parabolic problems," The Mathematics of Finite Elements and Applications III, MAFELAP 1978 (J. R. Whiteman, Ed.), Academic Press, New York, 1979, pp. 289-304.

10. Todd DuPONT \& Ridgway Scott, "Polynomial approximation of functions in Sobolev spaces," Math Comp., v. 34, 1980, pp. 441-463.

11. R. P. Fedorenko, “A relaxation method for solving elliptic difference equations," $\check{Z}$. Vycisl. Mat. i Mat. Fiz., v. 1, 1961, pp. 922-927.

12. R. P. Fedorenko, "The speed of convergence of one iterative process," Ż. Vycisl. Mat. i Mat. Fiz., v. 4, 1964, pp. 559-564.

13. P. GrisvarD, "Behavior of the solutions of an elliptic boundary value problem in a polygon or polyhedral domain," Numerical Solution of Partial Differential Equations-III (B. Hubbard, Ed.), Academic Press, New York, 1975, pp. 207-274.

14. W. НАсквUSCH, On the Convergence of a Multi-Grid Iteration Applied to Finite Element Equations, Report 77-8, Universität zu Köln, July 1977.

15. W. HАСквUSCH, On the Computation of Approximate Eigenvalues and Eigenfunctions of Elliptic Operators by Means of a Multi-Grid Method, Report 77-10, Universität zu Köln, August 1977.

16. P. JAMET, “Estimations d'erreur pour des éléments finis droits presque dégénérés," Rev. Francaise Automat. Informat. Recherche Operationelle, Ser. Rouge, v. 10, 1976, pp. 43-61.

17. R. A. Nicolaides, "On multiple grid and related techniques for solving discrete elliptic systems," J. Comput. Phys., v. 19, 1975, pp. 418-431.

18. R. A. Nicolaides, "On the $l^{2}$ convergence of an algorithm for solving finite element equations," Math. Comp., v. 31, 1977, pp. 892-906.

19. D. J. Rose \& G. F. WhitTEN, “A recursive analysis of dissection strategies," Sparse Matrix Computations (J. R. Bunch and D. J. Rose, Eds.), Academic Press, New York, 1976, pp. 59-83.

20. RIDGWAY SCOTT, "Interpolated boundary conditions on the finite element method," SIAM J. Numer. Anal., v. 12, 1975, pp. 404-427.

21. G. Strang \& G. Fix, An Analysis of the Finite Element Method, Prentice-Hall, Englewood Cliffs, N. J., 1973.

22. V. Tноме́е, "Negative norm estimates and super convergence in Galerkin methods for parabolic problems," Math. Comp., v. 34, 1980, pp. 93-113. 